\title{
Kosmoopera : entre Cosmos et Cosmogonie
}

\section{Kosmoopera: between cosmos and cosmogony}

\author{
Kristian Feigelson ${ }^{1}$ \\ ${ }^{1}$ Professeur des Universités - Université Sorbonne Nouvelle - Paris
}

RÉSUMÉ. L'exposition Kosmoopera est un projet de recherche qui a débuté en avril 2019. Un an après, on pourra découvrir les oeuvres de Valentina Mir au Centre de Russie pour la science et la culture à Paris. Cette exposition événement ouvrira ses portes le 7 avril 2020 à la veille de la fête nationale russe qui commémore le premier voyage de l'homme dans l'espace le 12 avril 1961. Grâce à l'amabilité et au soutien de Konstantin Volkov, le directeur du CRSC, Valentina Mir a pu fréquenter l'Institut et rencontrer à plusieurs reprises les cosmonautes G. Padalka et A. Borisenko. Ce dernier sera présent lors du vernissage et tiendra une conférence à cette occasion.

ABSTRACT. The Kosmoopera exhibition research project started in April 2019. A year later, the Russian Center for Science and Culture in Paris invite you to discover the works of Valentina Mir. The exhibition event take place on April 7, 2020, a few days before the Russian National Day, which commemorates the first human journey into outer space. Thanks to the kindness and support of Konstantin Volkov, chairman of the CRSC, Valentina Mir joins the Institute and meet the cosmonauts G. Padalka and A. Borisenko on several occasions. A. Borisenko will be present at the opening and will hold a conference.

MOTS-CLÉS. Valentina Mir, miramorphoses, kosmoopera, collage, Gagarine, Padalka, Borisenko, cosmonaute, Konstantin Volkov, CRSC.

KEYWORDS. Valentina Mir, miramorphoses, kosmoopera, collage, Gagarine, Padalka, Borisenko, cosmonaute, Konstantin Volkov, CRSC.

D'Europe jusqu'en Russie, Valentina Mir perpétue aujourd'hui à Paris son itinéraire d'artistevoyageuse du Cosmos. Le prisme de son regard initial s'élargit dans son Kosmoopera au-delà des frontières. Ici cet espace d'infni déploie des visions étonnées pour révéler un Cosmos inédit, inventif et expressif. A partir d'un processus de collage/recomposition, Valentina Mir nous propulse dans ce nouvel inconnu qui au départ nous semblait familier mais nous échappe. Cette mise en scène de l'espace comme des espaces pourrait rappeler les techniques russes du photo-montage. Mais son travail participe aussi d'une forme de décadrage. Elle nous propose en fait une invitation à un voyage bien plus vaste dans le Cosmos, alternant formes et fonds, conciliant le mouvement et l'espace-temps, la couleur et le noir et blanc. Rythmée de signes mystérieux, de références toujours plus colorées mais aussi fragmentées, faites de traces et d'empreintes dynamiques, issues des différents supports médias utilisés, cette œuvre composite nous invite à toute une série de rencontres interstellaires. Son Cosmos renvoie tant à la nostalgie de territoires nouveaux à conquérir que de territoires finalement inaccessibles. En explorant l'espace-temps de ces cosmonautes toujours plus aux confins de la terre et du ciel, Valentina Mir transgresse leurs frontières pour les rendre possibles et visibles. Sa démarche ancrée dans cette aventure soviétique du Cosmos peut alors se concevoir comme un nouveau récit de voyage hors du temps. Mais comment appréhender ce nouveau voyage utopique vers le Cosmos? Dans une démarche initiale qui pourrait nous rappeler le film soviétique muet «Aelita » de Protazanov 1924, lorsque la conquête de Mars apparaissait comme la métaphore décriée d'une utopie nouvelle. Mais ici, sa galaxie plus personnelle se revisite à partir de figures spatiales mélangées où les vaisseaux nous envelopperaient pour nous emporter vers de nouvelles fantasmagories. Ces Miramorphoses sont entre ciel et terre, entre imaginaire et réalité, entre photographie et peinture, entre verticalité et horizontalité. Elles dessinent des strates d'images diverses empilées ou juxtaposées, mêlant Mark Rothko et Valentina Terechkova, Alexeï Léonov et Vera Moukhina, Tatline et Lénine, Strelka avec Belka ...Elles irriguent et vivifent en permanence ce Cosmos réinventé comme lieu de passage personnel, entre droites, courbes, 
surfaces et profondeurs. Ses processus de collage s'ils révèlent en apparence une simplicité à la fois stylistique et ludique, dissimulent sur le fond une recherche sur l'image toujours personnelle et exigeante. Celle d'un Cosmos en suspension qui deviendrait au fur et à mesure la quête personnelle de sa propre Cosmogonie. Comme si cette recherche oscillant autour de ce mouvement perpétuel des images, nous invitait autant à un travail associatif qu'à une méditation sur l'infini. Les représentations accolées et collées de ces Mirs deviennent tout un kaleïdoscope d'impressions éclatées, renouvelées. Elles participent en permanence à un processus de métamorphoses des images. Celles-ci deviennent inépuisables, rappelant leurs sources multiples et démultipliées. Mais ces images sont autant d'histoires, décryptant les relations entre mémoires collectives et individuelles. La texture de ces images rassemblées, ne fait pas que révéler le passé mythique et glorieux de ces cosmonautes soviétiques, conquérants d'un inutile mais semble retranscrire leurs gestes, leurs voix, leurs confidences, leurs inquiétudes à vouloir toujours explorer cet infini. En redescendant sur terre avec Vostok 1, Youri Gagarine figure tutélaire, décrivait ces transformations : "Le vaisseau spatial était entouré de flammes,...j'étais un nuage de feu qui fonçait vers la terre! ». Des paroles déjà prophétiques qui lui conféreront dans l'éternité un statut d'astre terrestre, tour à tour astéroïde puis ange céleste bien que proclamant ne pas y avoir rencontrer son Dieu ! D'ailleurs, en conclusion Youri Gagarine propose dans son autobiographie "Chemin du Cosmos» (1961) sa propre vision pacifique de l'espace déclinant le Monde (Mir) sous l'angle de la Paix (Mirou) «Mir Mirou : nous faisons tout pour la paix!». Sans oublier que cette aventure spatiale soviétique s'était déroulée à l'époque dans un contexte de tensions internationales et de guerre froide, le Cosmos devenant un champ de compétitions nouvelles pour gagner la suprématie de l'espace. Ces paroles s'avèrent apporter un clin d'œil rétrospectif aux tableaux de Valentina Mir, nous proposant sa vision pacifiée et artistique du Cosmos. Ici les images se relient sous forme d'un récit. Derrière chaque histoire, il y aurait une part latente de Cosmos. Il ne s'agit pas de dépeindre un Cosmos abstrait mais de recréer un Cosmos plus intime où une image pourrait en cacher une autre. Participant d'une sorte d'histoire visuelle, Kosmoopera permet sous un mode ludique, de retrouver des images cachées pour en faire ressurgir d'autres. L'énergie de cet univers ne reflète pas la seule atmosphère de nos planètes mais nous offre la possibilité de partir dans un voyage de l'espace-temps autour de galaxie en mouvements. Aussi ces Miramorphoses, ne réhabilitent pas uniquement cette mémoire collective mais interpellent et réinventent ce Cosmos des promeneurs de l'espace. L'iconographie alors de Kosmoopera se redéploie comme une zone de virtualités nouvelles, dépassant l'épopée héroïque de la conquête de l'espace. Valentina Mir ne fait pas qu'illustrer tous ces points de passages pour recomposer en profondeur l'imagerie et l'imaginaire d'une épopée mythique. Conçu comme une véritable recherche, Kosmoopera participe à l'élaboration d'un nouveau mouvement vers l'infini. Son Cosmos devient une invitation nouvelle au voyage dans un cheminement toujours plus coloré à la croisée de différentes temporalités, celle d'hier et d'aujourd'hui. Mais ce voyage révèle aussi sa propre cosmogonie. Si Valentina Mir permet de reconstruire une vision nostalgique du Kosmoopera, nous invitant à l'accompagner ici dans de futurs périples, elle nous propose d'apprivoiser cette série de mystères cosmiques et d'y projeter nos propres imaginaires. 

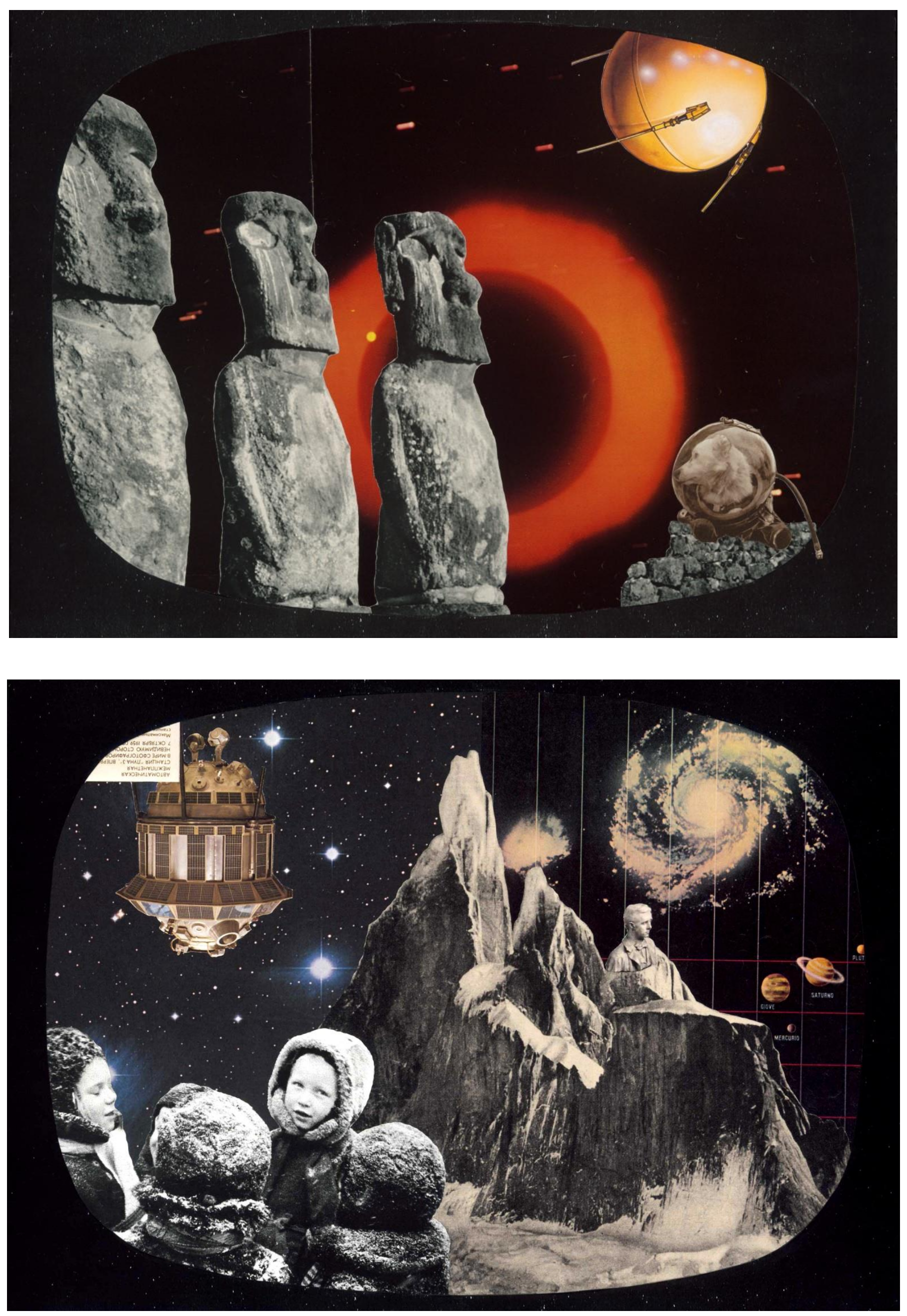

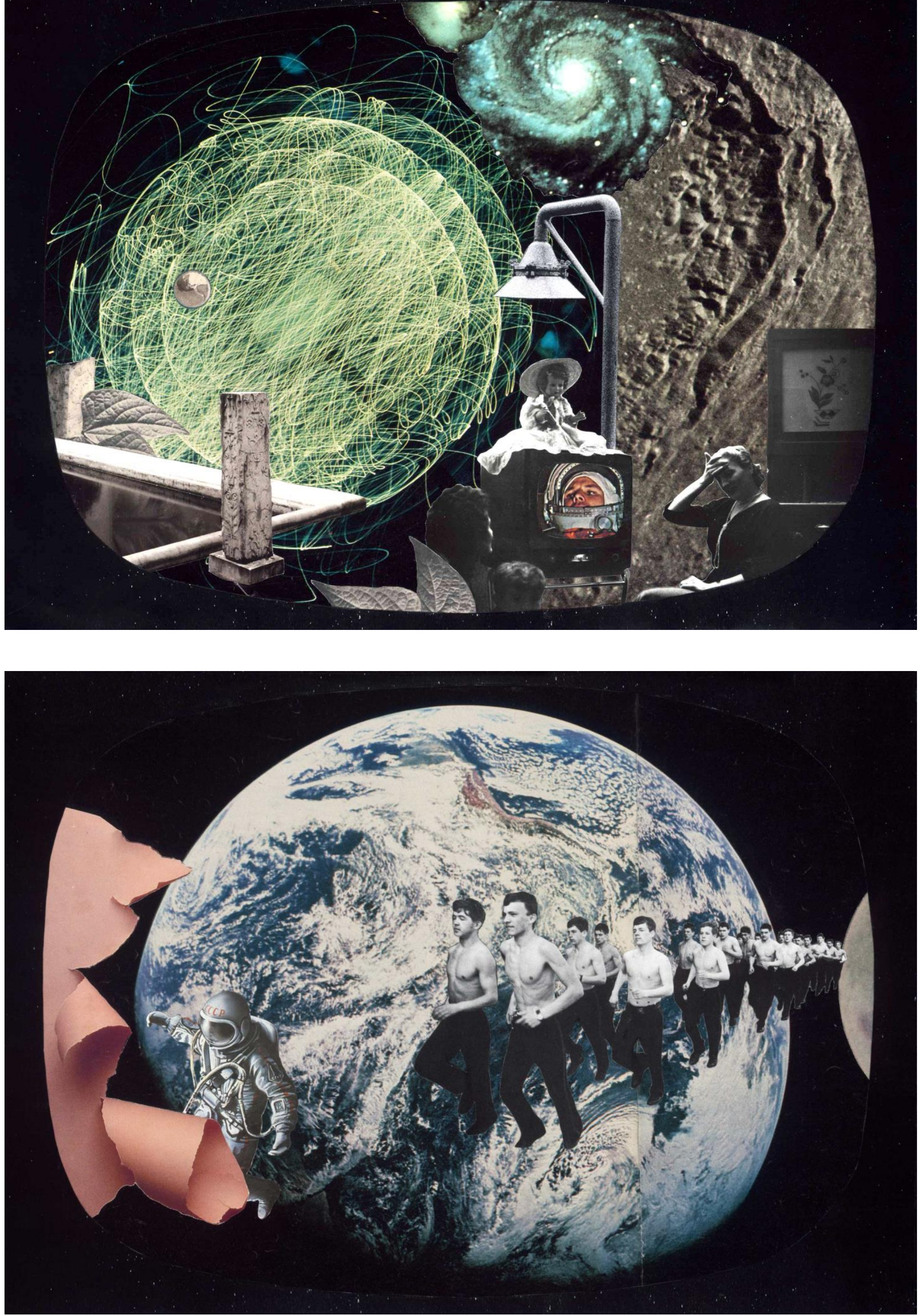

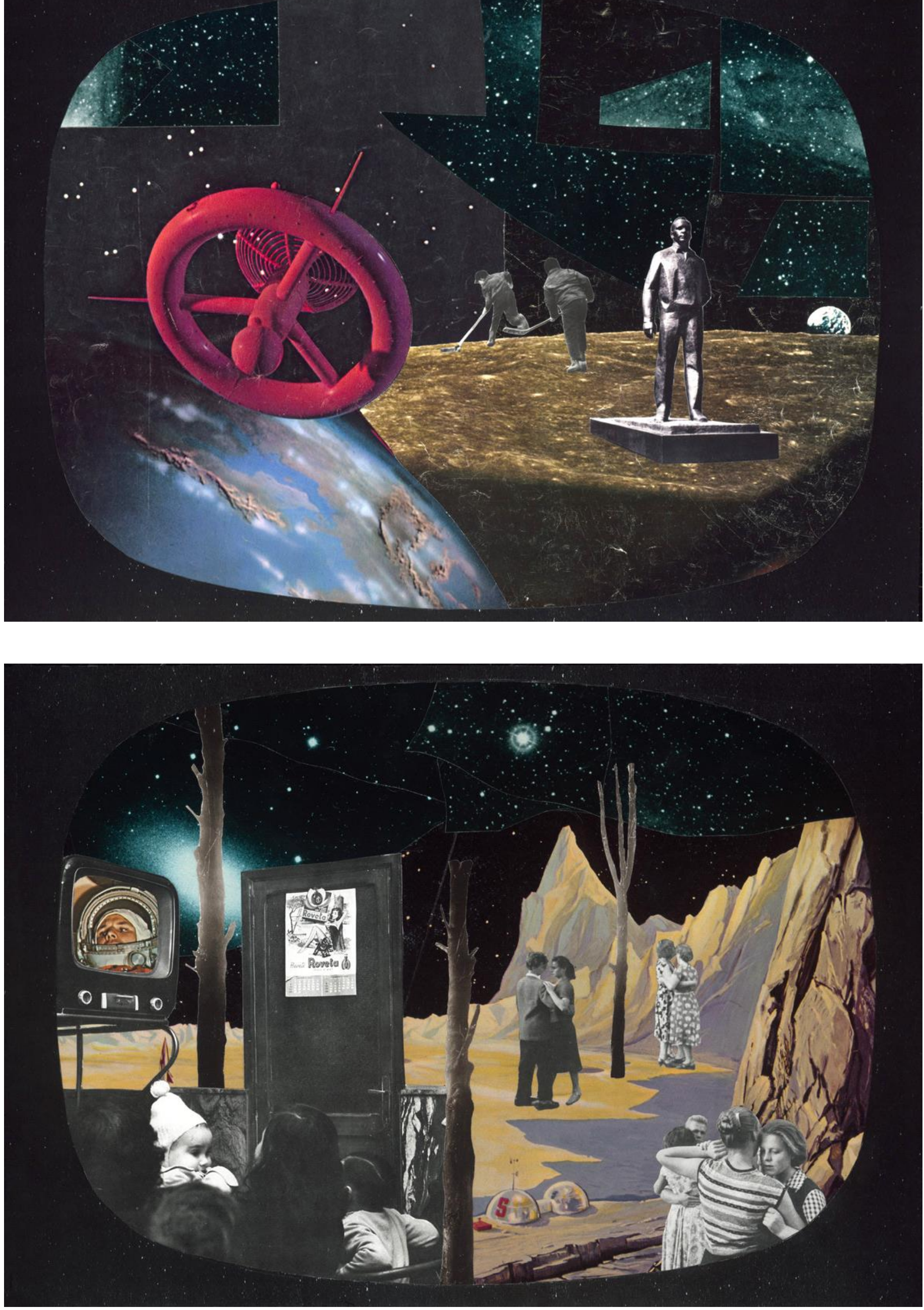


\section{Biographie}

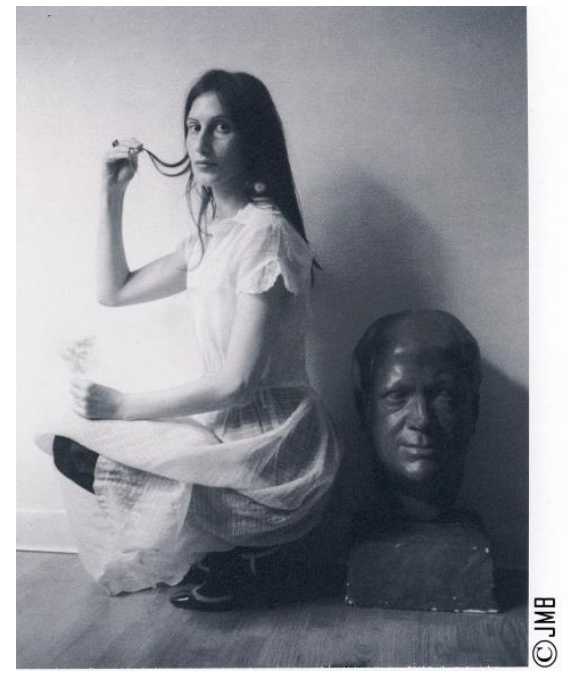

Valentina Mir est une artiste italienne qui vit et travaille à Paris. Ses oeuvres se situent entre l'analyse de la mémoire individuelle et l'étude de la façon dont cette mémoire devient imaginaire collectif.

\section{Les dates à retenir (sous réserve)}

\section{8 mars 2020.}

Vernissage de l'exposition collective: «Champ libre ». Valentina Mir y participe avec une installation immersive: Miramorphose KOSMOOPERA. Commissaire d'exposition Raphael Ocampo. L'exposition se poursuivra jusqu'au 31 Août 2020. Centre d'Art La Chapelle, 78120 Clairefontaine-en-Yvelines, France. Info: 01.34.94.39.87

\section{7 avril 2020.}

Vernissage de l'exposition personnelle KOSMOOPERA. Centre de Russie pour la science et la culture à Paris. L'exposition se poursuivra jusqu'au 12 Avril 2020. L'exposition a lieu dans le cadre de l'anniversaire de la cosmonautique liée à l'exploration de l'espace. CRSC, 61 rue Boissière, 75016 Paris. Réservation : crsc.paris@crsc.fr.

\section{Juillet 2020.}

Vernissage de l'exposition personnelle KOSMOOPERA. Quadrifoglio, Galleria d'Arte Contemporanea, Ortigia. L'exposition se poursuivra jusqu'au 31 Juillet 2020. Via dei Santi Coronati, 13, 96100 Siracusa SR, Italie. Info: 0039093164443 